\title{
Navegar contra el viento... O las perspectivas de América Latina en la era de la información ${ }^{1}$
}

\author{
Fernando Calderón*
}

\begin{abstract}
Resumen
El presente ensayo constituye una síntesis de las principales tendencias encontradas en una investigación relativa a multiculturalismo, desarrollo y política. Se realizaron 11 estudios nacionales, además de compartir y discutir un mapa cognitivo de la problemática; se impulsaron cuatro temas de investigación íntimamente vinculados tanto por la capacidad de acción de los actores del desarrollo como por las condiciones estructurales y los procesos históricos particulares de cada país. Se estudiaron las dinámicas del desarrollo económico y su articulación con el desarrollo humano, por una parte, y se vincularon ambos tipos de desarrollo con las transformaciones tecnoinformacionales y culturales que experimentó cada país. Asimismo, se asumió que la fuerza de las diversas orientaciones políticas y los conflictos socioculturales en cada uno de ellos organizaron las matrices de desarrollo mencionadas. El estudio no alcanzó todos los casos latinoamericanos y del Caribe que ameritan semejante pretensión, pero se avanzó hacia un panorama relativamente representativo, por cierto incompleto. Asimismo se tiene que tomar en cuenta que los procesos son nacionalmente distintos y una comprensión precisa necesita remitirse a los estudios de caso propiamente tales. Se trata de una realidad nacional diversa que comparte empero una serie de problemas, desafíos y opciones comunes.
\end{abstract}

Hay tres cuestiones que se trataron de conjugar en el presente estudio: el papel central del conflicto social y del poder en el desarrollo humano, el carácter condicionante del desarrollo informacional, y el rol central que juega en ellos la idea cultural de una vida digna. La vida digna se interpretó como la indivisibilidad de los derechos humanos y como fundamento de la libertad y la justicia y en consecuencia del desarrollo humano. La dignidad fundamenta la idea misma de libertad real. Ella sería un sustento de los valores de vida, por tanto de sostenibilidad intercultural del desarrollo.

Palabras clave: Desarrollo - multiculturalismo - conflicto social.

\begin{abstract}
This paper is a synthesis of the main trends found in a research on multiculturalism, development and politics. Eleven national studies were conducted, besides sharing and discussing a cognitive map of the problem; four research issues were promoted, all of them closely linked both by the capacity of action of development actors and by the particular historical processes of each country. The dynamics of economic development and its relationship with human development were studied, and both types of development were linked with techno-informational and cultural changes experienced by each country. It is also assumed that the strength of the various political
\end{abstract}

1 El presente texto se basa en el libro América Latina en la era de la información (dos tomos, Ediciones UNSAM). Allí se desarrollaron estudios de caso de Argentina, Bolivia, Brasil, Chile, Colombia, Costa Rica, México, Nicaragua, Panamá, Venezuela y Uruguay, además de reflexiones teóricas sobre la temática. Un equipo de investigadores de distintas universidades de América Latina, coordinados por Fernando Calderón desde la UNSAM en Argentina, realizó la investigación. Manuel Castells participó en seminarios con críticas y sugerencias.

* Coordinador del Programa sobre Innovación, Desarrollo y Multiculturalismo, Universidad Nacional de San Martín (UNSAM), Argentina. 
orientations and socio-cultural conflicts in each one of them organized their development models. The study did not reach all Latin American and Caribbean cases that warrant such a claim, but moved toward a relatively representative picture, certainly incomplete. Also it must be taken into account that processes are nationally different and that an accurate understanding needs to refer to the case studies. It is a diverse national reality that nevertheless shares a number of problems, challenges and common options.

There are three issues combined in this study: the central role of social conflict and power in human development, the conditioning character of informational development and the central role played in them by the cultural idea of a dignified life. The dignified life was interpreted as the indivisibility of human rights and as the foundation of freedom and justice and consequently of human development. The dignity underlies the very idea of real freedom. It would be a sustenance of life values, therefore of intercultural sustainability of development.

Keywords: Development - multiculturalism - social conflict.

\section{INTRODUCCIÓN}

¿Tendrán chances los diversos y desiguales procesos de desarrollo en curso en la región de lograr transformar los logros y limitaciones experimentados en la última década en fuerzas que les permitan sortear con éxito la crisis y las trasformaciones del capitalismo global, y así expandir e innovar dinámicas de un desarrollo humano de carácter cada vez más autónomo, incluyente e informacional? ¿Qué condiciones políticas e institucionales existen para promover y expandir un desarrollo humano informacional? ¿Qué actores pueden impulsarlo y cómo pueden construir regímenes democráticos legítimos y efectivos? En síntesis, ¿cuál es el nuevo sentido posible del desarrollo?

Para tratar de responder estas preguntas se realizaron 11 estudios nacionales, además de compartir y discutir un mapa cognitivo de la problemática; se impulsaron cuatro temas de investigación íntimamente vinculados tanto por la capacidad de acción de los actores del desarrollo como por las condiciones estructurales y los procesos históricos particulares de cada país. Se estudiaron las dinámicas del desarrollo económico y su articulación con el desarrollo humano, por una parte, y se vincularon ambos tipos de desarrollo con las transformaciones tecnoinformacionales y culturales que experimentó cada país. Asimismo, se asumió que la fuerza de las diversas orientaciones políticas y los conflictos socioculturales en cada uno de ellos organizaron las matrices de desarrollo mencionadas. El estudio no alcanzó todos los casos latinoamericanos y del Caribe que ameritan semejante pretensión, pero se avanzó hacia un panorama relativamente representativo, por cierto incompleto.

\section{PERSPECTIVAS CONCEPTUALES}

La experiencia de la investigación descansó en la crítica de tres perspectivas conceptuales acerca de la política, el desarrollo y la cultura.

La primera perspectiva está vinculada a las teorías estructurales del desarrollo impulsadas por la CEPAL y muy particularmente por los trabajos de Prebisch y Medina Echavarría. En Hacia una teoría de la transformación, Prebisch no solo realiza una crítica al capitalismo periférico sino que también plantea las limitaciones de la ortodoxia neoclásica para 
interpretar la realidad y para impulsar políticas viables para el desarrollo. Enfatiza a lo largo de su obra conceptos clave como "progreso técnico", "patrones de demanda", "intercambios desiguales" y "particularidades del capitalismo latinoamericano". Martín Puchet et al. (2016), en su estudio sobre el caso mexicano, hacen un balance crítico de esta perspectiva económica del desarrollo.

Por su parte, Medina Echavarría planteó la cuestión de las condiciones sociológicas del desarrollo económico. Las necesarias conexiones entre el tipo de institucionalidad y el modelo de desarrollo suponen actores con capacidad de construir una relación fecunda entre ambas dinámicas. Con Medina Echavarría se inicia la "Escuela de la Sociología Política del Desarrollo", que más adelante será desarrollada por variados autores en la región como E. Faletto, F.H. Cardoso (particularmente en su libro Dependencia y desarrollo económico), A. Gurrieri, J.C. Torrez, J.L. Reyna, entre otros pertenecientes a toda una generación de sociólogos latinoamericanos. Para Medina Echavarría una ética del desarrollo supone a la vez una responsabilidad compartida y una cierta ética del consumo. Precisamente aquí descansa una de las limitaciones crónicas más importantes de la región: la debilidad de las élites dirigentes para impulsar regímenes democráticos que puedan consolidar un desarrollo socialmente incluyente.

La idea movilizadora del desarrollismo cepalino supone un desarrollo "desde dentro" a partir de iniciativas del Estado, hacia una industrialización sustitutiva y hacia una modernización socialmente incluyente. Esta perspectiva sigue constituyendo un referente importante para comprender las nuevas opciones de desarrollo en los países de la región. "Vivir con lo nuestro", lo denomina hoy Aldo Ferrer.

En las décadas de los 80 y los 90, los trabajos de Fernando Fajnzylber reactualizaron este enfoque estructural del desarrollo, esta vez a base de una referencia comparativa con el sudeste asiático. Su artículo "Del hueco negro al casillero vacío" fue germinal. Las tesis referentes a la Transformación Productiva con Equidad (TPE) y atinentes al papel estratégico de la educación y el conocimiento en la misma TPE empezaron a colocar la gramática del estructuralismo en la temática de la globalización y el informacionalismo. Sin equidad no es posible un desarrollo competitivo y sostenible, argumenta el enfoque, que una vez más descansa en el progreso técnico, en la educación y en el conocimiento como llaves para vivir tiempos modernos. Incluso la CEPAL promovió un enfoque cultural del desarrollo que planteaba terminar con una cultura de la "negación del otro" y con arcanas estructuras de estratificación de clase de origen colonial o neocolonial, tan arraigadas en nuestras sociedades. Se buscó poner acento en asumir un tejido intercultural propio de las identidades múltiples y dinámicas de la región, promoviendo asimismo una ciudadanía activa como principal garantía de la TPE. Desgraciadamente los aspectos políticos de la TPE no pudieron estudiarse por barreras y circunstancias políticas limitativas que vivía la institución y la región. Ya eran los tiempos reinantes del neoliberalismo.

La segunda perspectiva está asociada a los informes relativos a desarrollo humano y política producidos sobre todo en Latinoamérica e influenciados por la perspectiva conceptual de Amartya Sen y Mabul UI Haq. Ambos, además de promover un enfoque interdisciplinario y 
empírico del desarrollo, impulsaron una visión holística, crítica, empírica y normativa del mismo, en el que la gente es el objeto y el sujeto del desarrollo.

Los conceptos de "libertad real", "capacidades" y "funcionamientos" son centrales en el pensamiento de Sen, así como los de "libertad cultural" y "agencia". Ellos son referencias importantes del enfoque del estudio. Para Sen el desarrollo supone mayor libertad. Su principal aporte fue vincular la idea de libertad con la idea de capacidad. Ser libre de tener hambre, de pasar enfermedades, de ausencia de conocimiento, de poder hablar en público, ser parte de relaciones sociales. De no tener pajcha, se diría en Cochabamba. En quechua, pajcha significa pobreza, que se comprende como no tener amigos, no tener lazo social; es decir, la pobreza está asociada a lo subjetivo, a los vínculos sociales y familiares y no solo a lo económico. Ser libre entonces para desarrollar las capacidades y elegir el tipo de vida que se sueña vivir y además tener el derecho a cambiar. Ser parte de colectividades, en nuestro enfoque, por elección personal y optar por lógicas individuales como resultado de deliberaciones colectivas.

Aquí vale la pena resaltar la importancia crucial que le da Sen a la relación entre cultura y desarrollo. Para él las conexiones son múltiples e insoslayables. La cultura, argumenta, es constitutiva del desarrollo, influencia en el comportamiento económico y produce remuneración. Está asociada con la participación política y la solidaridad social y es fundamental en la formación de valores (Sen, 2004). En breve, es lo que le da sentido al desarrollo y a la vida misma. Por eso, en sociedades multiculturales como las nuestras resulta fundamental fortalecer la idea de interculturalidad y la convivencia entre distintos con planos comunes de igualdad. Y esto está directamente asociado con el tema del conflicto, el poder y el cambio. Temas que ninguno de estos autores trabajó directamente y que para nosotros están en el centro de las disputas por el tipo de desarrollo que se busca.

Precisamente, la tercera perspectiva analítica está ligada a Alain Touraine y Manuel Castells, dos intelectuales que trabajan esta cuestión -e históricamente han estado vinculados al pensamiento del desarrollo en la región-, y a sus teorías de la acción y del poder que elaboraron desde distintos ángulos. En el libro La palabra y la sangre (no traducido del francés al español sino escrito en español en América Latina y luego traducido al francés), Touraine elabora un balance fundamental para entender los modos de desarrollo y los cambios sociopolíticos en Latinoamérica. Allí, las formas de poder en el proceso histórico del desarrollo están directamente asociadas con las orientaciones y las prácticas de los actores y el Estado. El concepto de campo de historicidad resulta crucial para comprender la dinámica del conflicto y el cambio en la actualidad (Touraine, 1973). Su penúltimo texto, Le fin de sociétés (El fin de las sociedades), plantea que la ética es el último recurso fundacional de un proyecto emancipatorio frente a una crisis multiglobal impuesta por las dinámicas antiéticas del capital financiero transnacional.

Por otra parte, el peso de los tres tomos de La era de la información (1999) de Manuel Castells y, más recientemente, su libro Comunicación y poder (2009), fueron cruciales para la estructuración conceptual y analítica a lo largo del estudio. La discusión y los seminarios dedicados a estas obras fueron permanentes, pues nos permitín vincular la globalización, 
la situación y las posibilidades de un desarrollo humano informacional en Latinoamérica, y con ello repensar las preguntas que nos hicimos al inicio del estudio.

En este ámbito, hay dos referencias analíticas que deseo particularmente destacar y que constituyen en realidad antecedentes directos de nuestro estudio. Por una parte, la discusión que tuvimos a inicios del presente siglo con un grupo de investigadores de la región acerca del sentido que tenían para Latinoamérica los cambios en la economía y la política global, tomando como referencia los tres tomos mencionados de Castells. El resultado fue un diálogo con él y un libro que se organizó con una pregunta acerca de si era sostenible la globalización en América Latina (Calderón, 2003). Eran los tiempos del fin del proceso neoliberal. Hoy habría que preguntarse, una vez más, si las experiencias neodesarrollistas son sostenibles frente a las necesidades de innovación y desarrollo y a los conflictos de poder en un momento de crisis global generalizada y bajo profundos cambios en los escenarios geopolíticos mundiales.

Por otro lado, un estudio referido a los modos de desarrollo en la era de la información coordinado por Manuel Castells y Pekka Himanen (2015) que analiza casos paradigmáticos -China, Sudáfrica, el Silicón Valley en Estados Unidos, Finlandia, Chile y Costa Rica-, además de un conjunto de trabajos teóricos relativos al tema, no solo fueron una referencia para nuestro trabajo sino en alguna medida una continuación de esa aventura.

Conceptualmente hay tres cuestiones que se trataron de conjugar en el presente estudio: el papel central del conflicto social y del poder en el desarrollo humano, el carácter condicionante del desarrollo informacional, y el rol central que juega en ellos la idea cultural de una vida digna. La vida digna se interpretó como la indivisibilidad de los derechos humanos y como fundamento de la libertad y la justicia y en consecuencia del desarrollo humano. La dignidad fundamenta la idea misma de libertad real. Ella sería un sustento de los valores de vida, por tanto de sostenibilidad intercultural del desarrollo. Una hipótesis es que las actuales protestas y movimientos socioculturales que suceden más o menos a nivel global, al buscar un nuevo sentido de la política para solucionar sus problemas de vida, estarían colocando en el centro del desarrollo una demanda de dignidad y redefiniendo así el campo histórico de lo posible.

En este ámbito, la idea de innovación es multidimensional y se refiere a la capacidad creativa de los actores individuales y colectivos en los distintos aspectos del desarrollo humano informacional, pero a partir de su constitución esencialmente multicultural. Como mencionamos en las notas teóricas del libro, el desarrollo informacional es un proceso de desarrollo que se corresponde con una lógica de acumulación capitalista una vez que el modelo de desarrollo industrialista previo entró en crisis. No se trata de la emergencia natural o necesaria en una línea de evolución de las sociedades y sus economías, sino de un "modelo" que pudo construirse dadas ciertas circunstancias históricas -económicas, políticas, culturales, tecnológicas- específicas. Cabalmente, la disputa por las orientaciones del informacionalismo en el periodo analizado entre orientaciones "neoliberales" y "neodesarrollistas", con todas sus variaciones, constituye el campo de historicidad de la época actual. 
Vale la pena retomar algunas referencias conceptuales:

El desarrollo informacional motorizó los procesos de globalización y estos, a su vez, lo impulsan. El desarrollo informacional se basa en la producción e interconexión de empresas, personas y organizaciones en redes que tienen la particularidad de poder actuar en tiempo real desde cualquier lugar conectado del planeta. Se basa en el uso de las tecnologías de información y comunicación, sin las cuales sería imposible el desarrollo moderno. No reemplazó al modelo de desarrollo industrial, sino que lo incorporó progresivamente a su lógica. Hoy los procesos de industrialización en América Latina para ser sostenibles necesitan integrarse a la dinámica informacional. Y ahí precisamente está uno de los nudos de la cuestión del desarrollo actual.

Además, innovar, como argumenta Castells, implica agregar un nuevo valor a un producto o servicio o al proceso de producción, consumo o distribución del mismo. La posibilidad de generar un nuevo valor se vincula con la capacidad creativa, que es la fuente que produce nuevo conocimiento. Aquí radicaría uno de los elementos fundamentales en la actualización del enfoque del desarrollo humano. La expansión de las capacidades creativas en el informacionalismo y en sus múltiples dimensiones son las que permiten impulsar un nuevo tipo de desarrollo humano: el desarrollo humano informacional.

La innovación, entonces, no solo es tecnológica; ella se produce a nivel social, organizacional y particularmente cultural. La innovación se vincula, además, a las capacidades sociales, culturales, educacionales, de agencia, de los actores individuales y colectivos, por lo que resulta fundamental el contexto (político, institucional, tecnológico, económico, ecológico, cultural, etc.) en el que la creatividad individual y social se desarrolla, como también la expansión y el manejo de los códigos de modernidad en el sentido elaborado en el texto Educación y conocimiento: eje de la TPE, y de los códigos del informacionalismo tratados en las notas teóricas del libro que permitió esta síntesis y que se refieren a poder navegar con creatividad los conflictos en las redes.

Asimismo, como la comunicación es central en los procesos creativos, se vuelve un aspecto clave en las posibilidades de generar innovación en los distintos ámbitos. Y ello tanto en los resultados de dichos procesos como en los procesos en sí mismos. Sin embargo es en el plano de la multiculturalidad donde la innovación cobra sentido. Un nuevo modo de vida posible y deseable centrado en la dignidad de las personas sería el que crearía otro tipo de desarrollo, lo que lógicamente vuelve a colocar a las orientaciones culturales de los actores en el centro de los conflictos de poder y de creatividad humana.

En breve, el triángulo de interacciones entre producción material, bienestar social y organización sociocultural sería el núcleo que produce desarrollo. Como afirman Castells y Himanen (2014: 29): "La competitividad de los territorios y firmas pasó a depender fuertemente de su habilidad para seguir las reglas de la economía informacional: creación de valor transformando información en conocimiento, y luego aplicación del conocimiento a todas las tareas a ser ejecutadas sobre la base de la capacidad tecnológica y humana incorporada en el sistema". Se daría una suerte de sinergia entre informacionalismo y productividad entre 
países, regiones y firmas, bajo dos condiciones: cambio organizacional bajo la forma de redes y mejora de la calidad del trabajo humano sobre la base de la educación y la calidad de vida. Sin embargo este informacionalismo se subordinó a un capitalismo financiero dominante que llevó a la crisis mundial actual y con ello a una crisis multicultural que redefine el orden y las posibilidades de desarrollo en la globalización. Esta ha sido precisamente la coyuntura histórica del estudio que aquí se sintetiza.

\section{TENDENCIAS GENERALES}

Inicialmente, se rescata la idea de que en la región las dinámicas del desarrollo son consecuencia tanto de los cambios globales en la era de la información como de conflictos sociales y de orientaciones políticas determinadas. La democracia de ciudadanía sería una condición necesaria del desarrollo y de la misma reinvención política de la sociedad y sus actores. Se entiende el desarrollo humano como la conflictiva construcción no de una sociedad perfecta, sino de una sociedad mejor, donde el bienestar conviva con la dignidad de las personas y las colectividades, y se postula un desarrollo humano innovador en los distintos componentes del desarrollo: competitividad, sostenibilidad, equidad, institucionalidad e interculturalidad, centrados en la capacidad de creación y agencia de la misma sociedad, donde la cultura y sus actores constituyen la mejor energía para avanzar en una sociedad de bienestar digna.

1. Orientaciones políticas diversas. Coexisten en la última década, foco del análisis realizado, orientaciones políticas diversas y cambiantes, que van desde opciones de modernización conservadora, como en el caso de Colombia y Panamá -donde el desarrollo y el cambio informacional son sobre todo el resultado de la fuerza del mercado y de valores tradicionales de conservación de un patrimonio nacional de las élites en el poder-, hasta orientaciones de neodesarrollismo comunitario o indígena en Bolivia, donde se busca conjugar lógicas entre un nuevo poder estatal como eje del desarrollo y la innovación y lógicas comunitarias de pueblos originarios de los Andes y la Amazonía, combinación por cierto alimentada por conflictos y visiones a menudo distintas y a veces contrapuestas.

Entre ambos extremos se desarrollan algunas combinaciones particulares de opciones de reformismo práctico de carácter neodesarrollista y con marcados rasgos nacionalpopulares como Venezuela, Nicaragua y, de manera más atenuada, Argentina y Brasil. También encontramos otras combinaciones de reformismo práctico, de tipo socialdemócrata, que gestionan modelos liberales como en Chile, Costa Rica y en cierta medida Uruguay. Ciertamente, las orientaciones políticas han cambiado en varios casos y los énfasis políticos también. Por otro lado, es posible reconocer una tendencia de recesión económica con fuerte impacto político y social, sobre todo asociada con los precios de las materias primas de exportación, pero también con el incremento de problemas institucionales y de carácter ético en el conjunto de las orientaciones políticas del desarrollo estudiadas, que debilitan o dificultan los intereses de las orientaciones mencionadas, además de otros factores claves para superar barreras y lograr un desarrollo humano informacional incluyente y, sobre todo, para enfrentar una multicrisis producida por un capitalismo financiero global que desestructura sociedades y regímenes democráticos en todas partes del mundo. 
2. Los niveles de desarrollo económico en la región son desiguales, como también los indicadores de desarrollo humano (véase Nota 1). Asimismo, los límites de un desarrollo informacional son evidentes y generalizados en todas las sociedades y economías de la región. Sin embargo, la región logró avanzar en la consolidación de un régimen democrático (los niveles de legitimidad de la democracia electoral son altos), crecer de manera sostenida en la última década, mejorar los indicadores de pobreza e incluso disminuir la desigualdad. No fueron pocos los logros de integración social, sobre todo bajo las orientaciones neodesarrollistas, y el Estado recuperó, limitadamente, un papel activo en la gestión del desarrollo y la política (véanse Notas 2, 3 y 4). Los avances en varios casos de una democracia participativa y de una mejor convivencia intercultural son evidentes, sin negar empero las notables dificultades en las distintas sociedades para expandir un pluralismo democrático. En el periodo analizado sobresalen nuevos problemas vinculados a la vida cotidiana referidos a la inseguridad, la economía perversa, la corrupción, el malestar, la frustración social, la legitimidad institucional, la contaminación ambiental y, sobre todo en algunos países, a la violación de los derechos humanos. También persisten patrones de desigualdad territorial entre campo y ciudad que se han reproducido e incluso en varios casos amplificado. Todos estos problemas son condiciones duras para cualquier apuesta política democrática que sea renovadora del desarrollo (véase Nota 5).

3. "Extractivismo informacional". Existirían avances en la economía tecnoinformacional, sobre todo en lo que se denominó preliminarmente como "extractivismo informacional", economía extractiva sustentada en inversiones novedosas y a veces muy creativas tecnológicamente para explotar los recursos naturales de exportación, pero también, en menor medida, de industrias de bienes secundarios vinculadas al mercado interno, regional o internacional. A partir de esta economía, el Estado ha jugado un rol central construyendo en varios casos una amplia coordinación económica y social, logrando estabilidad en las cuentas fiscales, inversiones estatales amplias y diversificadas, sobre todo en infraestructura; heterodoxas formas de asociación con nuevos capitales transnacionales para fortalecer tal "extractivismo"; y complejas ecuaciones políticas de un nuevo regionalismo latinoamericanista, aunque más político que económico o sociocultural.

4. Redes informacionales de comunicación. La incorporación social al mercado de las TIC es diferenciada a lo largo de la región, pero constante y creciente, como también lo es la expansión de industrias y mercados culturales de comunicación altamente monopólicos y de extraordinario poder cultural, político y económico. El consumo de internet y otros medios masivos de comunicación tiende a ser generalizado, y es mayor en la medida que los indicadores de desarrollo humano son más altos. En este ámbito, el Estado ha jugado un papel fundamental al crear plataformas de uso, consumo y educación en sistemas informacionales de acceso creciente y a veces priorizando a los sectores más excluidos, como es el caso del Plan CEIBAL, analizado en el caso de Uruguay, y los programas de entrega de computadores gratuitos en varios países de la región. El Estado mismo se ha modernizado informacionalmente en varios de sus aparatos de gestión burocrática, aunque el carácter patrimonial, corporativo y parainstitucional de las relaciones entre Estado y sociedad sigue siendo central a lo largo y ancho de Latinoamérica (véase Nota 6). 
5. Bajo desarrollo en ciencia y tecnología. Las inversiones en ciencia y tecnología son, en general, muy bajas. Los países con menor crecimiento registran aun menor inversión en investigación y más importaciones de alta tecnología y un incremento considerable del consumo de internet y derivados. El sistema de innovación es acorde con el patrón y nivel de crecimiento. El resultado es una baja tasa de inversión en investigación, ciencia y tecnología, y el desarrollo informacional innovador se canaliza predominantemente hacia la incorporación de alta tecnología en productos de exportación de origen primario. Sin embargo, vale la pena subrayar que si bien se ha avanzado en la incorporación de valor tecnológico local a los productos de exportación, gran parte de este tipo de contenido continúa siendo importado. Los avances en minería, petróleo, gas y agricultura han sido extraordinarios, pero también hubo una acelerada destrucción del medioambiente que viene deteriorando la sustentabilidad ecológica, por cierto muy a menudo afectando territorios y lógicas comunitarias de pueblos originarios en regiones como la Amazonía en Sudamérica (véase Nota 7).

6. Concentración, pobreza y desigualdad. Bajó la pobreza, y también la desigualdad en menor escala, pero se mantuvieron los niveles de concentración de riqueza. La tesis de una complementariedad entre igualdad y competitividad en el sentido de la TPE supone que la equidad predispone a la sostenibilidad de un desarrollo económico siempre y cuando las capacidades políticas de los actores y el Estado puedan complementar de manera compleja avances combinados y pertinentes entre una dinámica y otra. En esta cuestión, los avances han sido insuficientes pero ya están instalados en el horizonte de las sociedades como tareas pendientes.

7. El sistema de innovación es congruente con un patrón de crecimiento predominantemente "extrovertido". El resultado final, sobre todo comparado con el sudeste asiático, es una baja tasa de inversión en investigación y desarrollo. El ingreso per cápita en periodos largos no crece y la igualdad en la distribución del ingreso crece poco. Sin embargo, la disminución de la pobreza, sobre todo la absoluta, ha sido impresionante prácticamente en toda la región. La cuestión es el tipo, la cualidad y la sostenibilidad de la inserción de los excluidos. Posiblemente aquí radique uno de los desafíos futuros fundamentales del conjunto de los países de la región (véase Nota 8).

8. Asincronía cultural. Retomando el concepto de "asincronía" de Germani (1966) para explicar los procesos de cambio esta vez a nivel multicultural, es posible concluir de los estudios realizados que los sentidos, la dirección y la intensidad del cambio cultural se han complicado y muestran potencialidades de cambio inéditas en la región. Así, resaltan las experiencias de reconocimiento y construcción institucional a nivel de las relaciones interétnicas y culturales; un ejemplo en este sentido es Bolivia, donde se creó una nueva Constitución que transformó el Estado, el sistema político y las lógicas culturales, reconociendo el rol central de los pueblos originarios en la conformación del Estado y de la democracia; asimismo, se impulsaron políticas antidiscriminatorias, pero sin resolver la construcción plena y práctica de una interculturalidad plural, sobre todo en los ámbitos urbanos y en la Amazonía. Estos procesos han estado cruzados por tensiones y conflictos aun no resueltos, como se destacó en el estudio del caso boliviano. En el otro extremo están el "modelo 
de modernización conservadora" en Colombia y la crisis multicultural mexicana que, bajo la apariencia de un reconocimiento multicultural, al final re-significan un modelo monocultural históricamente dominante. En medio de estos casos existen muy sugerentes avances, aunque no suficientes, en la mayoría de los casos estudiados. En el plano de los derechos humanos se destacan logros institucionales, sobre todo en Argentina, en especial en relación con la memoria y la justicia; en ese mismo país también hubo avances notables en el reconocimiento institucional de plenos derechos referidos a los migrantes. Por otro lado, es particularmente destacable la búsqueda de construcción de un proceso de paz en Colombia que tal vez condicione una apertura democrática y de cambio en los patrones de desarrollo. Su resultado, sobre todo si es positivo para la paz, afectaría no solo a la región sino a los conflictos multiculturales a nivel mundial.

En el plano de la industria y el mercado cultural los cambios han sido considerables en toda la región. Un relativamente nuevo poder cultural, económico y político de los medios de comunicación tiende a estar muy concentrado en todos los países y constituye además una extraordinaria fuerza de la tecnoeconomía de la información. Su impacto en el consumo y la vida cotidiana de las personas es sobresaliente. Los sistemas y regímenes políticos predominantes en la región no alcanzan a procesar tal poder, por cierto con dinámicas internas a menudo contradictorias por la presencia de fuerzas y componentes disímiles. Sin embargo, ellos en gran medida redefinen las agendas políticas con sentidos ambivalentes. O Globo en Brasil, la red Clarín en Argentina o Televisa en México, son referentes regionales y globales. En gran medida su poder está asociado con la emergencia de una política y un nuevo poder de lo público, fenómeno complementario con el desarrollo de una nueva tecnosociabilidad que, entre otros factores, supone no solo el acceso creciente a los medios de comunicación masivos vinculados a internet y a la telefonía celular, sino muy particularmente a nuevas formas de acción colectiva e individual. Como se mencionó en el estudio La protesta social en América Latina, en su gran mayoría los conflictos sociales se desplazan para ser gestionados en la red y los nuevos movimientos socioculturales, como los de estudiantes en Chile en 2011, en México en 2012 y en Brasil en 2014, ejemplifican la emergencia de una nueva politicidad, entendida como nuevos sentidos de la política y como ampliación del campo de lo posible. El estudio de caso de la actual situación chilena es muy ilustrativo al respecto.

9. Desigualdades de género. Un tema que merece especial interés y que necesita ser estudiado mejor es el papel de las relaciones de género en el desarrollo, pues a pesar de importantes avances en el reconocimiento, la distribución y participación de las mujeres en el desarrollo en la última década, las situaciones estructurales de desigualdad persisten y atraviesan al conjunto de los procesos de desarrollo analizados. El sistema patriarcal, tan arraigado en las sociedades y culturas latinoamericanas, constituye un límite estructural del desarrollo humano informacional. Empero, el movimiento feminista está buscando redefinir el espacio público, renovando una democracia más deliberativa, donde la relación entre lo público, lo privado, la política y el desarrollo sea consociativa. Este tema es, sin lugar a dudas, la cuestión central de un cambio ético, político y cultural en la sociedad red emergente. 
En síntesis, la "asincronía" multicultural en todas sus dimensiones, aquí ultrasintetizadas, constituye la nueva dinámica del cambio sistémico vinculado a las nuevas formas de poder y, sobre todo, a una nueva construcción sociocultural de la dignidad que, con todas sus complejidades y realidades, ya emergió en la región.

10. Frustración de expectativas. Posiblemente, de no mediar cambios drásticos y positivos de políticas de desarrollo humano informacional incluyentes, estaremos frente a una creciente y nueva "frustración de expectativas" y a un creciente malestar social respecto de la política y sus instituciones. El valor agregado del sector TIC observa en casi todos los casos una tasa de crecimiento promedio anual mayor que los puestos de trabajo y las remuneraciones; si a esto se agrega el incremento acelerado de los consumos de TIC y de mejoría relativa de los niveles de educación y, con todo ello, mayores aspiraciones y expectativas de la sociedad, es muy posible que los Estados no tengan, sobre todo por las condiciones internacionales de recesión, posibilidades de satisfacerlas. Así, estaremos frente a un creciente malestar social que puede terminar minando y deslegitimando los logros y los procesos de desarrollo logrados, alimentando nuevos conflictos y opciones políticas inéditas. Cabalmente, las demandas de una nueva politicidad que registran los casos de Chile, México y Brasil, entre otros países, alimentarían esta tendencia (véase Nota 8). Hoy está en juego una suerte de redefinición del campo de historicidad.

11. La nueva y cambiante geopolítica. El cambio en el escenario geopolítico global asociado con la multicrisis en curso ya ha colocado a la región en medio de complicadas ecuaciones económicas, culturales y políticas. Aquí vale la pena resaltar dos aspectos. Por un lado, el reforzamiento de las relaciones financiero-comerciales, de inversión en infraestructura y productivas, especialmente de China, es transversal casi a todos los países de la región. Además se inician o renuevan dinámicas con India, Rusia, África y Asia, sin dejar de lado el peso estructural de las economías desarrolladas de occidente, en particular de Estados Unidos. Hoy el mundo es multipolar y de nuevo tipo y los latinoamericanos ya estamos navegando en él. No se sabe si se navegará como barco a la deriva o con estrategias y cartas de navegación hacia un desarrollo que reduzcan el riesgo y empujen al bienestar. Por otro lado, la crisis y la reestructuración económica global y la emergencia de diversos problemas socioinstitucionales en la mayoría de los países, colocan a las diversas apuestas de desarrollo, en especial a las políticamente progresistas, ante la disyuntiva de cómo enfrentar la crisis: si se hace reinventando ecuaciones políticas y económicas, ampliando y desarrollando una lógica informacional asociada con innovaciones productivas, políticas, institucionales y culturales, o rediseñando ajustes socioeconómicos que supongan costos sociales y de gobernabilidad política. Nuevamente, los temas de gobernabilidad de la economía, de umbrales mínimos de funcionamiento de las instituciones y de niveles o límites de la integración social y cultural, están en las opciones y escenarios políticos latinoamericanos.

\section{COROLARIO}

En el fondo, la cuestión consiste en cómo se puede mutar de las diferentes situaciones y procesos de dependencia que predominaron en el pasado hacia una mayor interdependencia 
y autonomía. Es decir, se trata de detectar si hay condiciones, voluntad y posibilidades políticas para promover, a escala regional y nacional, un desarrollo humano informacional genuino y sostenible. Finalmente, es posible concluir que los procesos de desarrollo en curso posibilitan una diversidad de interacciones que podría promover una mayor autonomía e independencia. Ello supone, empero, incrementar cualitativamente la capacidad de agencia de los actores del desarrollo de cada país y de la región en su conjunto.

Todavía no se aprende a navegar plenamente contra el viento, como argumentaba Hirschman, pero en eso estamos...

\section{BIBLIOGRAFÍA}

Araníbar, A. y B. Rodríguez (2013): "Latinoamérica, ¿del neoliberalismo al neodesarrollismo?", en América Latina, ¿del neoliberalismo al neodesarrollismo?, Proyecto de Análisis Político y Escenarios Prospectivos (PAPEP), PNUD, Siglo XXI, Buenos Aires.

Cardoso, F.H. y E. Faletto (2003): Dependencia y desarrollo en América Latina, Siglo XXI, Buenos Aires. Castells, M. (1999): La era de la información (volúmenes I, II y III), Siglo XXI, México D.F. (2009): Comunicación y poder, Alianza, Madrid.

Castells, M. y P. Himanen (eds.) (2014): Reconceptualizing development in the global information age, Oxford University Press.

Comisión Económica para América Latina y el Caribe (CEPAL) (2015a): Desigualdad, concentración del ingreso y tributación sobre las altas rentas en América Latina (editado por Juan Pablo Jiménez), CEPAL, Santiago de Chile.

(2015b): Desigualdad e informalidad. Un análisis de cinco experiencias latinoamericanas (editado por Verónica Amarante y Rodrigo Arim), CEPAL, Santiago de Chile.

(2015c): Bases de datos y publicaciones estadísticas CEPALSTAT, CEPAL, Santiago de Chile.

Fajnzylber, F. (1990): “Transformación productiva con equidad”, Libros de la CEPAL, 25.

(1992): "Industrialización en América Latina. De la 'caja negra' al 'casillero vacío'", Cuadernos de la CEPAL, 60.

Ferrer, A. (2001): Vivir con lo nuestro. Nosotros y la globalización, Fondo de Cultura Económica, Buenos Aires.

(2010): El futuro de nuestro pasado. La economía argentina en su Segundo Centenario (primera edición), Fondo de Cultura Económica, Buenos Aires.

Germani, G. (2010): Gino Germani. La sociedad en cuestión: antología comentada, Consejo Latinoamericano de Ciencias Sociales (CLACSO), Buenos Aires.

Medina Echavarría, J. (1980): La Obra de José Medina Echavarría, Ediciones Cultura Hispánica, Madrid.

Puchet, M., C. Zúñiga, J. Díaz y M. García (2016): "México ante la globalidad: interculturalismo, innovación y desarrollo como dignidad", en F. Calderón (coord.): America Latina en la era de la información (tomo II), en prensa.

Prebisch, R. (1980): Hacia una teoría de la transformación, CEPAL, Santiago de Chile.

Sen, A. (1999): Desarrollo y libertad, Planeta, Barcelona. 
(2004): "How does culture matter?", en V. Rao and M. Walton (eds.): Culture and public action, Stanford University Press.

Touraine, A. (1973): Production de la société, Editions du Seuil, Paris.

(1988): La parole et le sang, Odile Jacob, Paris.

Recibido: 24-08-2015

Aceptado: 31-08-2015 


\section{ANEXO: NOTAS EMPÍRICAS PARA LA ILUSTRACIÓN DE TENDENCIAS}

Las presentes notas solo ilustran las tendencias señaladas. Para una mayor verificación empírica véase el libro América Latina en la era de la información.

\section{Nota 1}

El cuadro que se presenta más abajo muestra los países bajo análisis agrupados según su nivel de desarrollo humano. Chile y Argentina son los únicos con un grado muy alto de desarrollo humano. Argentina posee la mayor cantidad de años esperados de escolaridad. Chile encabeza el ranking de años promedio de escolaridad. Asimismo, junto con Costa Rica, cuyo nivel de desarrollo humano es alto, tienen la mayor esperanza de vida al nacer.

En el Informe de desarrollo humano 2013 se calculó el IDH excluyendo los ingresos. Para todos los países, salvo Brasil y Colombia, la diferencia entre el ranking del IDH y el ranking del Ingreso Nacional Bruto per cápita (INB) era positiva. La mayor brecha entre ambos rankings se observó en Chile, Costa Rica y Uruguay. En los casos de Colombia y Brasil, esta diferencia era negativa, es decir, el INB tenía una mejor clasificación que su nivel de desarrollo humano.

Índice de Desarrollo Humano (IDH) y sus componentes, 2013

(INB ajustado por PPP en dólares constantes de 2011)

\begin{tabular}{|c|c|c|c|c|c|c|c|c|}
\hline RANKING & PAÍS & $\mathrm{IDH}$ & $\begin{array}{c}\text { ESPERANZA } \\
\text { DE VIDA AL } \\
\text { NACER }\end{array}$ & $\begin{array}{c}\text { AÑOS } \\
\text { PROMEDIO DE } \\
\text { ESCOLARIDAD }\end{array}$ & $\begin{array}{c}\text { AÑOS } \\
\text { ESPERADOS DE } \\
\text { ESCOLARIDAD }\end{array}$ & $\begin{array}{l}\text { INB PER } \\
\text { CÁPITA }\end{array}$ & $\begin{array}{l}\text { IDH } \\
2012\end{array}$ & $\begin{array}{l}\text { VARIACIÓN } \\
\text { EN EL } \\
\text { RANKING } \\
\text { IDH }\end{array}$ \\
\hline \multicolumn{9}{|c|}{ Desarrollo humano muy alto } \\
\hline 41 & Chile & 0,822 & 79,96 & 9,8 & 15,1 & 20.804 & 0,819 & 1 \\
\hline 49 & Argentina & 0,808 & 76,31 & 9,8 & 16,4 & 17.297 & 0,806 & 0 \\
\hline \multicolumn{9}{|c|}{ Desarrollo humano alto } \\
\hline 50 & Uruguay & 0,790 & 77,23 & 8,5 & 15,5 & 18.108 & 0,787 & 2 \\
\hline 68 & Costa Rica & 0,763 & 79,93 & 8,4 & 13,5 & 13.012 & 0,761 & -1 \\
\hline 71 & México & 0,756 & 77,50 & 8,5 & 12,8 & 15.854 & 0,755 & -1 \\
\hline 79 & Brasil & 0,744 & 73,94 & 7,2 & 15,2 & 14.275 & 0,742 & 1 \\
\hline 82 & Perú & 0,737 & 74,83 & 9,0 & 13,1 & 11.280 & 0,734 & 0 \\
\hline 98 & Colombia & 0,711 & 74,04 & 7,1 & 13,2 & 11.527 & 0,708 & 0 \\
\hline 98 & Ecuador & 0,711 & 76,47 & 7,6 & 12,3 & 9.998 & 0,708 & 0 \\
\hline \multicolumn{9}{|c|}{ Desarrollo humano medio } \\
\hline 111 & Paraguay & 0,676 & 72,26 & 7,7 & 11,9 & 7.580 & 0,670 & 0 \\
\hline 113 & Bolivia & 0,667 & 67,26 & 9,2 & 13,2 & 5.552 & 0,663 & 0 \\
\hline
\end{tabular}

Fuente: Elaboración propia sobre la base de datos del Informe de desarrollo humano 2014. 
¿Es sostenible la baja de la desigualdad en la región cuando se evidencian limitaciones significativas en los actuales modelos de desarrollo latinoamericanos bajo un persistente contexto económico internacional desfavorable?

Índice de Concentración de Gini. América Latina (promedio simple) (años 1997, 1999, 2002, 2005, 2008, 2010, 2012 y 2013)

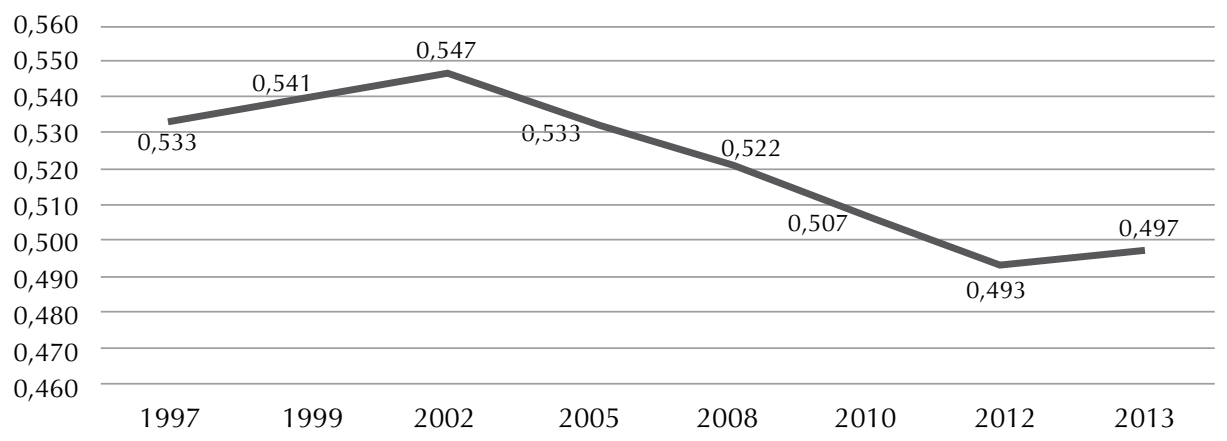

Fuente: Elaboración propia en base a datos de CEPAL (2015).

\section{Nota 2}

La expansión del empleo fue uno de los principales factores que incidieron en la mejora en los niveles de distribución del ingreso en la región durante el periodo considerado, así como también un eslabón fundamental en los procesos de integración social de los modelos de desarrollo analizados.

Tasa de desempleo. América Latina y el Caribe, 1991-2014

12.50

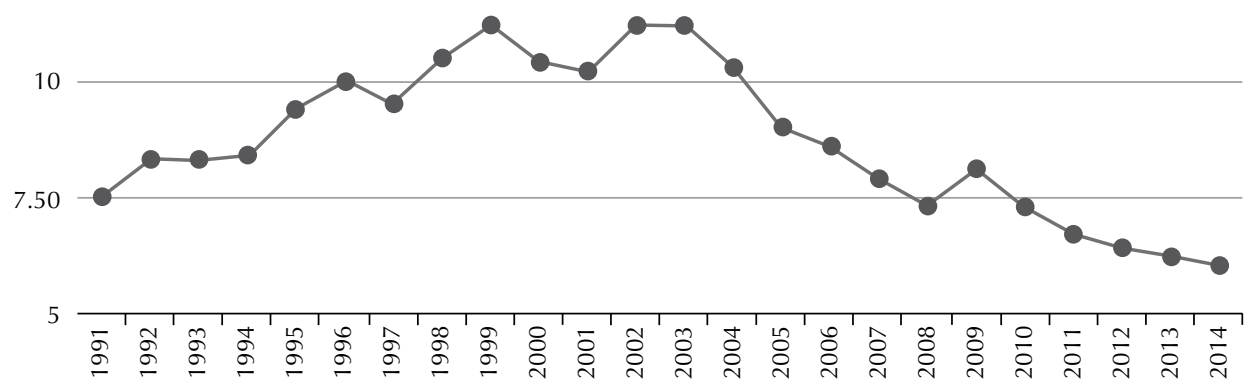

Fuente: CEPAL (2015). 


\section{Nota 3}

Luego de la crisis del final del periodo neoliberal, en la primera década del siglo se registra una baja generalizada en los niveles de desconfianza en las instituciones políticas y en el Estado en la región, donde se observan las particularidades históricas de cada caso. Ahora bien, en los últimos años la desconfianza vuelve a emerger, ahora bajo los ejes de nuevas demandas sociales e institucionales.

Porcentaje de desconfianza en las instituciones políticas y en el Estado. América Latina y el Caribe, 1996-2013

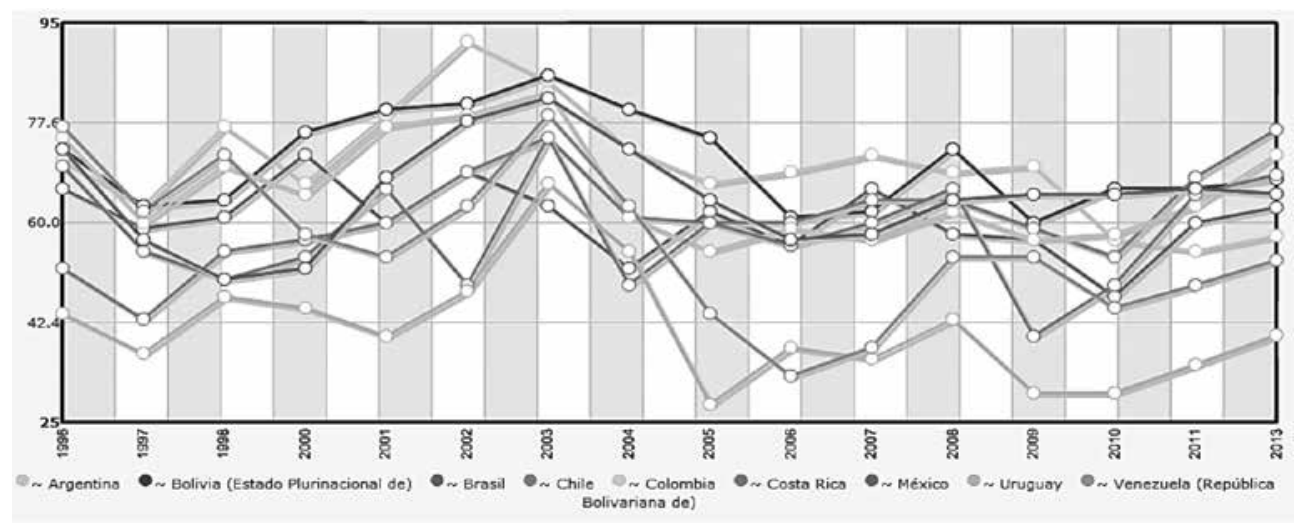

Fuente: CEPAL (2015).

\section{Nota 4}

Durante el primer decenio del siglo XXI los términos de intercambio registraron los niveles más favorables de los últimos 30 años para las economías de la región. En el último lustro los niveles siguen siendo altos en términos históricos, aunque registran una tendencia a la baja como consecuencia de los cambios producidos en los mercados internacionales a partir de la crisis internacional desatada en el año 2008.

Índice de términos de intercambio de bienes $(2010=100)$.

América Latina y el Caribe, 1980-2014

125

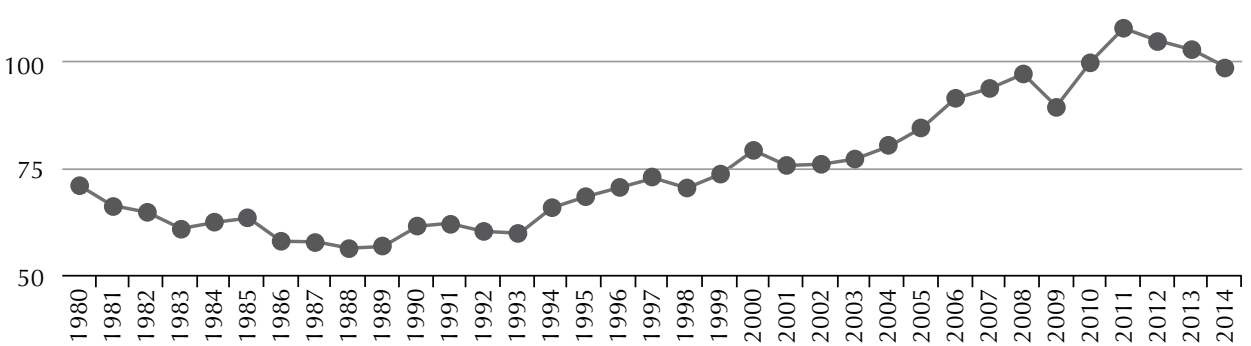

Fuente: CEPAL (2015). 


\section{Nota 5}

No sin cierta volatilidad, los niveles de inversión extranjera directa durante la segunda etapa del periodo analizado (2007-2014) alcanzaron los registros más altos de las últimas tres décadas en la región. La mayoría de las inversiones se volcó directa e indirectamente en proyectos basados en la extracción de recursos naturales, muchas veces bajo formas heterodoxas de asociación público-privadas.

Inversión extranjera neta (millones de dólares).

América Latina y el Caribe, 1990-2014

200000

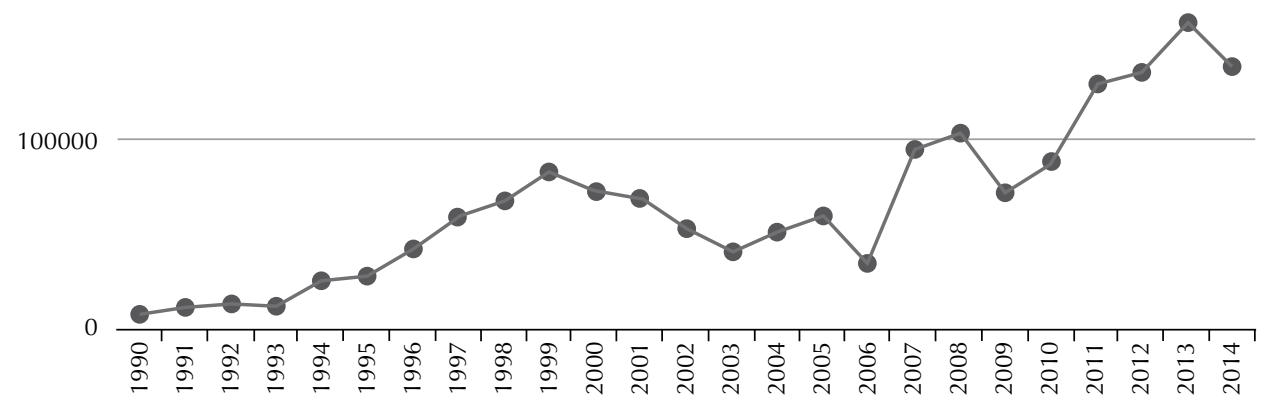

Fuente: CEPAL (2015). 


\section{Nota 6}

Brasil, México y Argentina concentran el 92\% de la inversión que se hace en América Latina y el Caribe en investigación y desarrollo (l+D). A pesar de lo avanzado en la última década, los países de la región se encuentran lejos de las mejores prácticas internacionales.

Relación porcentual entre los gastos de investigación y desarrollo respecto

del PBI en países seleccionados, año 2011

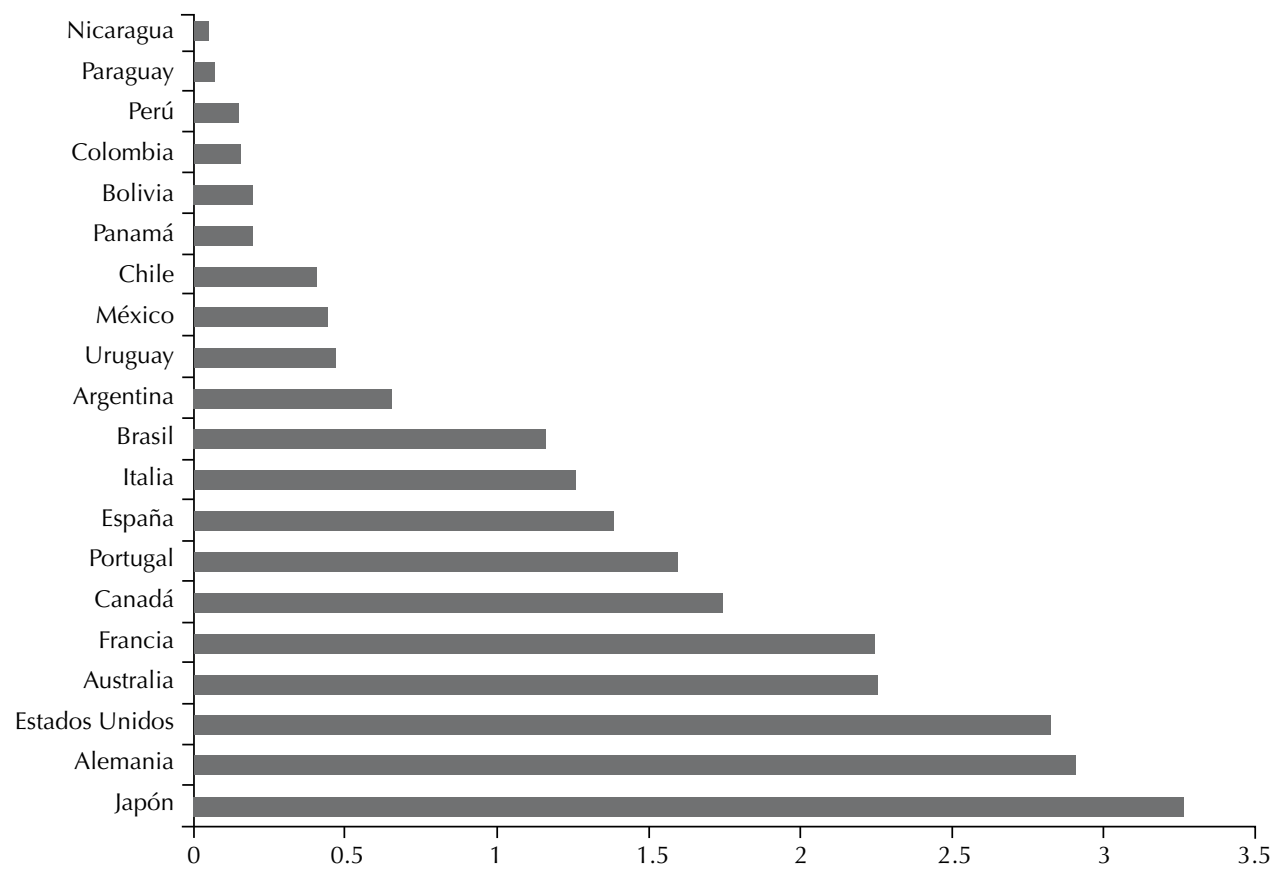

Fuente: "Indicadores de Ciencia y Tecnología, Argentina 2011", Ministerio de Ciencia, Tecnología e Innovación Productiva (2013).

\section{Nota 7}

Los países de la región han logrado sustanciales avances en el acceso a las TIC en los últimos 15 años, aunque se registran brechas digitales en relación con el género y estrato socioeconómico y entre países. 
Suscriptores de telefonía celular móvil y usuarios de internet en América Latina. Cantidad cada 100 habitantes

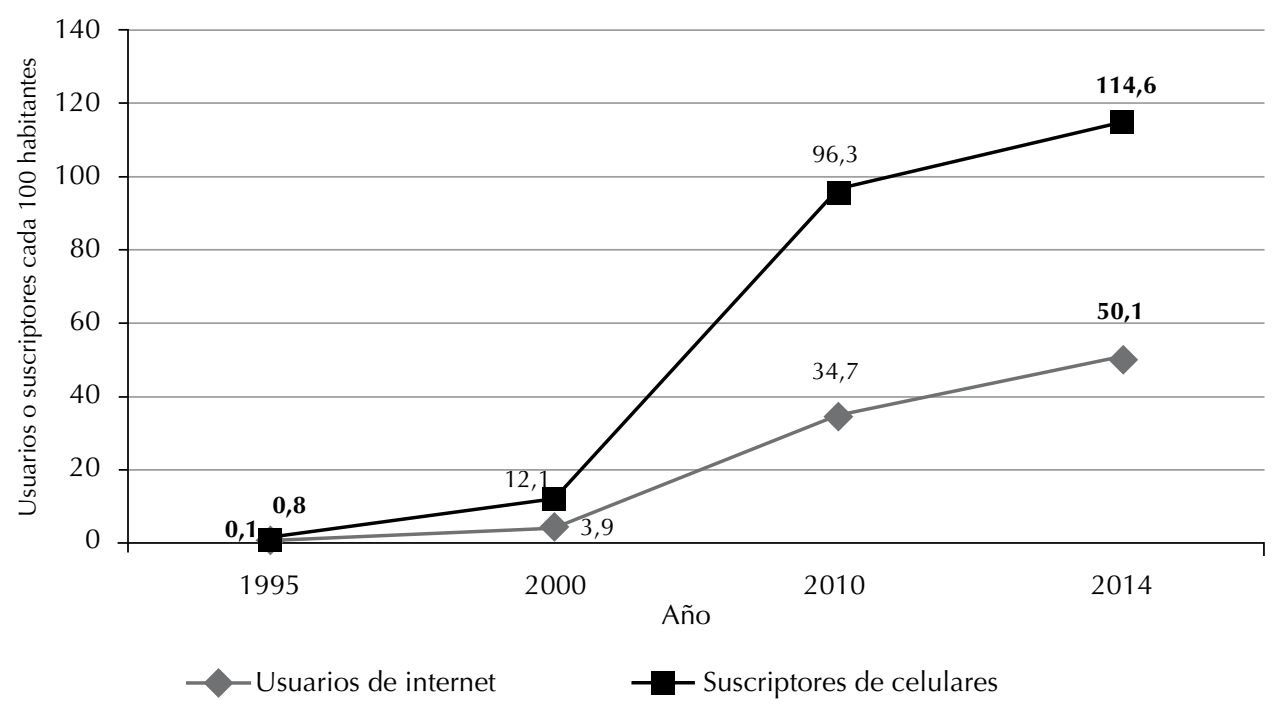

Fuente: CEPAL (2015).

\section{Nota 8}

Contrastando con la figura anterior, en términos de empleo, desempleo y participación la región muestra los indicadores más favorables de los últimos 20 años. Sin embargo, el salario real no ha aumentado significativamente si se lo compara con otros indicadores, máxime considerando que al 2013 prácticamente la mitad (46\%) de los ocupados urbanos de la región están empleados en el sector informal y 1 de cada 10 vive en hogares en situación de indigencia (CEPAL, 2015).

Salario Medio Real Anual (2000=100). América Latina, 1992-2013

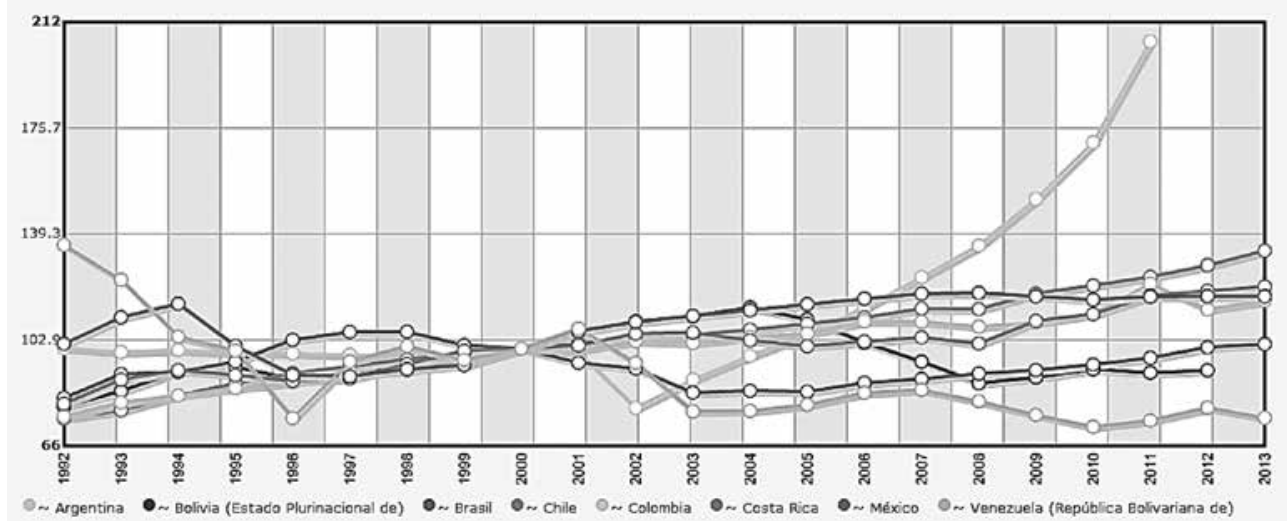

Fuente: CEPAL (2015). 